\section{AlH versus prontuário médico no estudo do risco de óbito hospitalar no infarto agudo do miocárdio no Município do Rio de Janeiro, Brasil}

\author{
Hospital admissions forms versus medical records \\ to assess risk of in-hospital death from acute \\ myocardial infarction in Rio de Janeiro, Brazil
}

\author{
${ }^{1}$ Hospital dos Servidores do \\ Estado, Ministério da Saúde, \\ Rio de Janeiro, Brasil. \\ 2 Escola Nacional de Saúde \\ Pública, Fundação Oswaldo \\ Cruz, Rio de Janeiro, Brasil. \\ 3 Núcleo de Estudos de Saúde \\ Coletiva, Universidade \\ Federal do Rio de Janeiro, \\ Rio de Janeiro, Brasil. \\ 4 Instituto Brasileiro \\ de Geografia e Estatística, \\ Rio de Janeiro, Brasil. \\ Correspondência \\ C. C. Escosteguy \\ Serviço de Epidemiologia, \\ Hospital dos Servidores do \\ Estado, Ministério da Saúde. \\ Rua Sacadura Cabral 178 \\ Rio de Janeiro, $R J$ \\ 20221-903, Brasil. \\ cescosteguy@hse.rj. \\ saude.gov.br
}

\section{Abstract}

The objective of this study was to analyze the usefulness of the Brazilian Hospital Information System (SIH) in comparison to medical records to study factors associated with in-hospital mortality due to acute myocardial infarction (AMI). We evaluated a stratified random sample of 391 medical records (out of 1,936 hospital admissions forms) with AMI as the primary diagnosis in the city of Rio de Janeiro. Factors associated with in-hospital death were studied through logistic modeling. Models were developed directly from the SIH and from medical records. ROC curves were constructed to allow comparison of the different models. We found an AMI diagnostic confirmation $=91.7 \%$ and hospital mortality $=20.6 \%$. The logistic model derived from medical records produced the best fit (concordance $=90.1 \%$ ). Although the SIH model had a worse fit (concordance $=70.6 \%$ ), the correction of keying-in and information errors using data from medical records did not significantly modify its performance. Underrecording of secondary diagnosis was high in the SIH forms and was the main limiting factor.

Information Systems; Myocardial Infarction; Hospital Mortality; Medical Records
Claudia Caminha Escosteguy 1 Margareth Crisóstomo Portela 2 Roberto de Andrade Medronho 1,3 Mauricio Teixeira Leite de Vasconcellos 4

\section{Introdução}

As doenças cardiovasculares têm sido a principal causa de mortalidade proporcional no Brasil desde a década de 60 , sendo responsáveis por 27,0\% do total de óbitos em 2000 (Ministério da Saúde. Sistema de Informações sobre Mortalidade. http://www.datasus.gov.br, acessado em 21/Ago/2002). A doença isquêmica do coração é o componente principal dessa mortalidade nas cidades das regiões Sul e Sudeste 1.

Dentre as doenças cardiovasculares, o infarto agudo do miocárdio é especialmente indicado para o desenvolvimento de indicadores e padrões de qualidade, considerando-se, por um lado, o impacto na mortalidade, a letalidade hospitalar e o volume de internações que acarreta, e por outro, a sua sensibilidade a tecnologias médicas hospitalares. O infarto agudo do miocárdio é um evento agudo que sempre requer internação hospitalar, constituindo um diagnóstico clínico relativamente simples e bem estabelecido, geralmente baseado no tripé história clínica, evolução eletrocardiográfica e curva enzimática 2,3 . Além disso, trata-se de uma condição ímpar do ponto de vista de disponibilidade de evidência científica, possibilitando que o uso de grande parte das tecnologias terapêuticas disponíveis seja baseado em numerosas publicações que documentam sua eficácia, segurança e custo.

Estudos têm mostrado grande variação no processo da assistência hospitalar ao infarto 
agudo do miocárdio, assim como na mortalidade hospitalar a ele associada. No Brasil, alguns estudos têm discutido fatores associados à variação do risco de óbito hospitalar associado ao infarto agudo do miocárdio e, menos freqüentemente, à qualidade dessa assistência hospitalar 4,5,6.

Nesse contexto, a existência de uma grande base de dados nacional sobre informações hospitalares - o Sistema de Informações Hospitalares do Sistema Único de Saúde (SIH/SUS) - mais conhecido como o "Sistema da AIH" (Autorização de Internação Hospitalar), representa um instrumento que merece ser avaliado enquanto uma fonte de dados passíveis de serem utilizados na avaliação da assistência hospitalar por ele coberta. O banco de dados da AIH tem sido utilizado em alguns estudos da variação do uso de recursos hospitalares, da morbidade e da mortalidade hospitalar 4,5,7,8,9. A revisão da literatura encontrou dois estudos sobre a confiabilidade dos dados da AIH no país, ambos no Estado do Rio de Janeiro. O estudo de Travassos-Veras \& Martins 10 concluiu ser alta a confiabilidade das variáveis sexo, idade e tempo de permanência, do diagnóstico principal em três dígitos da nona revisão da Classificação Internacional da Doença (CID-9) da Organização Mundial da Saúde (OMS) e dos procedimentos realizados. Artigo nosso 11 discutiu a pertinência do uso da AIH na avaliação da qualidade da assistência ao infarto agudo do miocárdio, documentando a qualidade satisfatória do diagnóstico de infarto agudo do miocárdio da AIH, assim como a precisão de variáveis demográficas (sexo, faixa etária), de processo (uso de procedimentos e intervenções) e de resultado (óbito, motivo da saída).

Tendo em vista essas considerações, o objetivo deste estudo é avaliar o desempenho do banco da AIH em relação ao prontuário médico na análise dos fatores associados à variação do risco de óbito hospitalar no infarto agudo do miocárdio, no Município do Rio de Janeiro, referente ao ano de 1997.

\section{Metodologia}

O universo do estudo foi definido com base no campo Diagnóstico Principal da AIH, que representa o diagnóstico que geralmente é preenchido à admissão, ou a suspeita inicial que motiva a internação. Na base de dados de 1997 é usada a CID-9; o diagnóstico de infarto agudo do miocárdio corresponde à CID-9 410.9/2. Inicialmente foi realizada uma análise exploratória do universo de $1.936 \mathrm{AIH}$ registradas com esse diagnóstico no Município do Rio de Janeiro em 1997. Em seguida, realizou-se um estudo seccional de uma amostra aleatória de 391 prontuários médicos, estratificada por hospitais, sorteada a partir desse universo.

O cálculo do tamanho da amostra levou em consideração a proporção de pacientes e óbitos, com um erro relativo de $10 \%$ e um nível de significância de 5\%, em um modelo de amostragem estratificada com alocação proporcional e sem reposição. Os pacientes foram sorteados baseando-se nos 22 hospitais incluídos na amostra; do universo inicial de 38 hospitais, foram excluídos aqueles que atenderam menos de dez casos no ano estudado. O trabalho de campo envolveu coleta de dados nos prontuários médicos para a confirmação do diagnóstico de infarto agudo do miocárdio, dados sobre fatores de risco e gravidade, e dados sobre o uso de intervenções diagnósticas e terapêuticas. O protocolo de estudo foi aprovado pela Comissão de Ética em Pesquisa da Escola Nacional de Saúde Pública da Fundação Oswaldo Cruz 6 .

O diagnóstico de infarto agudo do miocárdio foi confirmado segundo os critérios de caso definitivo ou possível adotados pelo Projeto MONICA da OMS 12. Esses critérios, resumidamente, envolvem história de dor torácica do tipo isquêmica, alterações eletrocardiográficas evolutivas obtidas em traçados seriados e elevação sérica de marcadores enzimáticos de necrose miocárdica seguida por sua queda. Com base na combinação desses critérios, os casos foram classificados em infarto agudo do miocárdio definitivo (presença de dois ou mais critérios ou evolução eletrocardiográfica de injúria e necrose miocárdica compatível com infarto agudo do miocárdio), infarto agudo do miocárdio possível (presença de quadro clínico sugestivo, porém sem critério eletrocardiográfico ou enzimático que confirme definitivamente o diagnóstico), infarto agudo do miocárdio afastado (quando os sintomas e os testes não permitem classificá-lo como infarto agudo do miocárdio confirmado/possível, ou quando o episódio agudo é explicado por outra afecção) e ignorado.

Realizou-se análise univariada e multivariada por meio dos programas Epi Info 6.04d e SAS ${ }^{\circledR}$ (versão 6.0 ). O teste do $\chi^{2}$ foi usado para testar a significância estatística de diferenças observadas nas proporções das categorias das variáveis estudadas; considerou-se estatisticamente significativo um valor de $\mathrm{p}<0,05$ bicaudal. Quando necessário foi usado o teste exato de Fisher. Foram calculados intervalos de confiança de 95\% (IC95\%) das proporções e razões 
de chance (OR, de odds ratio) estimadas. Para o estudo dos fatores associados à variação no risco de óbito hospitalar foi utilizada análise multivariada por regressão logística; neste caso, o teste de Wald foi usado para testar a significância das variáveis independentes incluídas no modelo. Para a análise do ajuste global do modelo utilizou-se o teste da razão de verossimilhança (-2 LOG L). Foi analisada a concordância entre as probabilidades preditas pelo modelo e respostas observadas; a presença de interação entre as variáveis; o diagnóstico da regressão por meio da análise dos resíduos e observações extremas; e o possível impacto dos mesmos sobre o modelo. Usando-se a modelagem hierárquica foi testado o efeito randômico no nível dos hospitais. Todas as variáveis incluídas nos modelos apresentados foram categóricas: a categorização da idade em faixas etárias permitiu um melhor ajuste, por se observarem, na amostra, pontos de corte bem caracterizados.

Foram construídas curvas ROC (Receiver Operator Characteristic) 13 para cada modelo estudado, a partir da mensuração da sensibilidade e especificidade para cada ponto de corte dos modelos. A área sob a curva ROC descreve a acurácia geral do modelo. Para comparar as curvas ROC correspondentes a dois modelos distintos e, conseqüentemente, o desempenho relativo entre eles, foi testada a significância estatística da diferença entre as duas áreas das curvas ROC correspondentes.

\section{Resultados}

Das 391 AIH sorteadas, a busca ativa dos prontuários médicos nos hospitais resgatou $384 \mathrm{ca}$ sos (perda de 1,8\%, não diferenciada entre óbitos e não óbitos). O diagnóstico atendeu os critérios de infarto agudo do miocárdio definitivo ou possível em 91,7\% de casos (IC95\%: 88,3$94,2)$, sendo $87,0 \%$ de infarto agudo do miocárdio definitivo (IC95\%: 83,1-90,1).

A Tabela 1 resume as principais variáveis categóricas disponíveis nas AIH utilizadas para o estudo do risco de morte na amostra de infarto agudo do miocárdio, informando também os valores correspondentes encontrados nos prontuários. A distribuição das variáveis estudadas segundo a AIH foi semelhante na base do município como um todo e na amostra selecionada ( $p>0,05)$. Já a distribuição e a letalidade associada a algumas variáveis se modificaram ao serem analisados os dados coletados do prontuário. Em geral, o uso de procedimentos foi sub-registrado na AIH em relação ao prontuário. A variável Diagnóstico Secundário corresponde ao campo da $\mathrm{AIH}$ que agrega qualquer outra CID informada além daquela correspondente ao diagnóstico principal, tendo sido muito sub-registrado.

A letalidade geral na amostra de prontuários foi de 79 óbitos, ou 20,6\% dos casos (IC95\%: 16,7-25,0). Segundo a AIH eram 77 óbitos; dois foram digitados como alta (ambos em hospitais municipais).

A Tabela 2 apresenta os resultados de dois modelos logísticos estudados com base nas variáveis existentes na $\mathrm{AIH}$. Os testes para efeito randômico não alcançaram significância estatística, optando-se, portanto, pela regressão logística clássica. O modelo 1 (original) utilizou os valores originais da $\mathrm{AIH}$, conforme digitados no banco. O modelo 2 (corrigido) usou os valores corrigidos a partir dos dados encontrados nos prontuários médicos. Tal correção permitiu avaliar o impacto sobre os modelos estudados dos problemas da qualidade da informação obtida diretamente do banco da AIH em relação àquela coletada a partir do prontuário revisto. Ambos os modelos usaram variáveis disponíveis na AIH. A diferença reside no fato de o modelo 2 corrigir as falhas de digitação e precisão do banco da AIH e o sub-registro do diagnóstico secundário.

A correção das variáveis digitadas na AIH aumentou a concordância do modelo, a significância estatística da menor chance de óbito associada aos casos que fizeram uso de cateterismo cardíaco e a significância estatística da maior chance de óbito associada à natureza universitária do hospital. Além disso, aumentou a razão de chances associada à presença de qualquer diagnóstico secundário e fez com que esta variável passasse a exibir significância estatística.

A Tabela 3 apresenta algumas variáveis de gravidade e de processo de assistência presentes apenas no prontuário médico, que foram incorporadas à modelagem. A Tabela 4 mostra o modelo estudado a partir do prontuário médico. Como não foi detectado efeito randômico significativo, optou-se pela regressão logística clássica. O teste de Hosmer-Lemeshow (resumido pela estatística ĉ na Tabela 4) indicou um ajuste satisfatório para cada decil de probabilidade estimada de óbito, a partir da comparação das freqüências observadas e esperadas em cada decil.

Faixas etárias mais elevadas associaram-se a uma maior chance de óbito do que a categoria de referência de 60 anos ou menos, de forma consistente, em todos os modelos estudados. As variáveis relativas aos fatores de risco 
Variáveis categóricas da AlH utilizadas no estudo do risco de morte na amostra de infarto agudo do miocárdio no Município do Rio de Janeiro, Brasil, em 1997, e distribuição correspondente segundo o prontuário.

\begin{tabular}{|c|c|c|c|c|c|c|}
\hline \multirow[t]{2}{*}{ Variável } & \multicolumn{3}{|c|}{$\mathrm{AlH}$} & \multicolumn{3}{|c|}{ Prontuário médico } \\
\hline & $f$ & $\%$ & $\begin{array}{c}\text { Letalidade } \\
\text { (\%) }\end{array}$ & $f$ & $\%$ & $\begin{array}{c}\text { Letalidade } \\
\text { (\%) }\end{array}$ \\
\hline \multicolumn{7}{|l|}{ Sexo } \\
\hline Masculino (categoria de referência) & 238 & 62,0 & 16,4 & 236 & 61,5 & 17,8 \\
\hline Feminino & 146 & 38,0 & 26,0 & 148 & 38,5 & 25,0 \\
\hline \multicolumn{7}{|l|}{ Idade (em anos) } \\
\hline$\leq 60$ (categoria de referência) & 190 & 49,5 & 12,6 & 187 & 49,0 & 12,8 \\
\hline $61-80$ & 176 & 45,8 & 23,9 & 180 & 47,1 & 25,6 \\
\hline$>80$ & 18 & 4,7 & 61,1 & 15 & 3,9 & 53,3 \\
\hline \multicolumn{7}{|l|}{ Uso de UTI (ou UC) } \\
\hline Com UTI (categoria de referência) & 227 & 59,1 & 18,5 & 312 & 81,3 & 19,2 \\
\hline Sem UTI & 157 & 40,9 & 22,3 & $72^{\star}$ & 18,8 & 18,1 \\
\hline \multicolumn{7}{|l|}{ Uso de trombolíticos } \\
\hline $\operatorname{Sim}$ & 32 & 8,3 & 21,9 & 75 & 19,5 & 13,3 \\
\hline Não (categoria de referência) & 352 & 91,7 & 19,9 & $309 *$ & 80,5 & 22,3 \\
\hline \multicolumn{7}{|l|}{ Uso de cateterismo cardíaco } \\
\hline Sim & 20 & 5,2 & 5,0 & 51 & 13,3 & 2,0 \\
\hline Não (categoria de referência) & 364 & 94,8 & 20,9 & $333^{*}$ & 86,7 & 23,4 \\
\hline \multicolumn{7}{|l|}{ Uso de angioplastia coronária } \\
\hline $\operatorname{Sim}$ & 4 & 1,0 & 0,0 & 8 & 2,1 & 12,5 \\
\hline Não (categoria de referência) & 380 & 99,0 & 20,3 & $376^{*}$ & 97,9 & 20,7 \\
\hline \multicolumn{7}{|l|}{ Uso de revascularização miocárdica } \\
\hline $\operatorname{Sim}$ & 5 & 1,3 & 40,0 & 4 & 1,0 & 25,0 \\
\hline Não (categoria de referência) & 379 & 98,7 & 19,8 & $380 *$ & 99,0 & 20,5 \\
\hline \multicolumn{7}{|l|}{ Diagnóstico secundário } \\
\hline Sim (qualquer um presente) & 43 & 11,2 & 25,6 & 326 & 84,9 & 21,2 \\
\hline Não (categoria de referência) & 341 & 88,8 & 19,4 & 58 & 15,1 & 17,2 \\
\hline \multicolumn{7}{|l|}{ Natureza jurídica } \\
\hline Estadual & 85 & 22,1 & 22,4 & 85 & 22,1 & 22,4 \\
\hline Federal & 116 & 30,2 & 19,8 & 116 & 30,2 & 19,8 \\
\hline Municipal (categoria de referência) & 140 & 36,5 & 16,4 & 140 & 36,5 & 17,9 \\
\hline Privada & 12 & 3,1 & 33,3 & 12 & 3,1 & 33,3 \\
\hline Universitário & 31 & 8,1 & 25,8 & 31 & 8,1 & 25,8 \\
\hline
\end{tabular}

* Incluídos uso não realizado ou não informado (ignorado). 
Tabela 2

Risco de óbito hospitalar na amostra de infarto agudo do miocárdio a partir dos valores digitados na AlH (modelo 1) e dos corrigidos pelo prontuário (modelo 2).

\begin{tabular}{|c|c|c|c|c|c|c|}
\hline \multirow[t]{2}{*}{ Variável } & \multicolumn{3}{|c|}{ Original da AlH (Modelo 1) } & \multicolumn{3}{|c|}{ Corrigido (Modelo 2) } \\
\hline & OR & IC95\% & $\begin{array}{c}\operatorname{Pr}>\chi^{2} \\
P\end{array}$ & OR & IC95\% & $\begin{array}{c}\operatorname{Pr}>\chi^{2} \\
P\end{array}$ \\
\hline Intercepto & - & - & 0,0001 & - & - & 0,0001 \\
\hline Feminino & 1,5 & $0,8-2,5$ & NS & 1,2 & $0,6-2,1$ & NS \\
\hline \multicolumn{7}{|l|}{ Faixa etária (em anos) } \\
\hline $61-80$ & 2,1 & $1,2-3,7$ & 0,02 & 2,2 & $1,2-3,9$ & 0,009 \\
\hline+ de 80 & 10,3 & $3,5-30,6$ & 0,0001 & 7,5 & $2,3-24,3$ & 0,0009 \\
\hline Uso de UTI & 0,9 & $0,4-1,5$ & NS & 0,7 & $0,3-1,5$ & NS \\
\hline Uso de trombólise & 1,2 & $0,4-3,4$ & NS & 0,7 & $0,3-1,5$ & NS \\
\hline Uso de cateterismo cardíaco & 0,1 & $0,01-1,2$ & 0,07 & 0,03 & $0,003-0,3$ & 0,003 \\
\hline Uso de revascularização miocárdica & 6,6 & $0,5-91,8$ & NS & 7,3 & $0,3-177,8$ & NS \\
\hline Diagnóstico secundário & 1,4 & $0,6-3,4$ & NS & 2,4 & $1,3-4,3$ & 0,002 \\
\hline \multicolumn{7}{|l|}{ Natureza jurídica } \\
\hline Privado & 2,8 & $0,7-3,4$ & NS & 2,4 & $0,5-10,8$ & NS \\
\hline Estadual & 1,5 & $0,7-3,3$ & NS & 1,4 & $0,7-3,0$ & NS \\
\hline Federal & 1,5 & $0,7-2,9$ & NS & 1,4 & $0,7-2,8$ & NS \\
\hline Universitário & 2,5 & $0,9-7,0$ & 0,08 & 4,8 & $1,6-15,0$ & 0,006 \\
\hline$\chi^{2}$ para covariáveis* & \multicolumn{3}{|c|}{35,6 com $12 \mathrm{GL}(p=0,0002)$} & \multicolumn{3}{|c|}{$57,1 \operatorname{com} 12 \mathrm{GL}(p=0,0001)$} \\
\hline Concordância & \multicolumn{3}{|c|}{$70,6 \%$} & \multicolumn{3}{|c|}{$75,6 \%$} \\
\hline Teste de Hosmer-Lemeshow & \multicolumn{3}{|c|}{$\hat{c}=5,80$ com 8 GL $(p=0,67)$} & \multicolumn{3}{|c|}{$\hat{c}=5,76 \operatorname{com} 8 \mathrm{GL}(p=0,67)$} \\
\hline
\end{tabular}

* Estimado a partir da diferença entre -2LOG L do intercepto apenas e do intercepto + covariáveis.

Tabela 3

Variáveis de gravidade e processo de assistência encontradas apenas na amostra de prontuários.

\begin{tabular}{|c|c|c|c|c|c|c|c|}
\hline Variável do prontuário & $f$ & $\%$ & IC95\% & Letalidade (\%) & $O R^{*}$ & IC95\% & $p$ \\
\hline \multicolumn{8}{|l|}{ Classe Killip à admissão } \\
\hline । & 317 & 82,6 & $78,3-86,1$ & 12,3 & 1,0 & - & - \\
\hline II & 19 & 5,0 & $3,1-7,8$ & 42,1 & 5,2 & $1,8-15,0$ & 0,002 \\
\hline III & 9 & 2,3 & $1,1-5,2$ & 55,6 & 8,9 & $2,0-41,7$ & 0,003 \\
\hline IV & 11 & 2,9 & $1,5-5,2$ & 90,9 & 71,3 & $9,0-1.524,0$ & 0,000000 \\
\hline Não descrita & 28 & 7,3 & $5,0-10,5$ & 60,7 & 11,02 & $4,5-27,4$ & 0,000000 \\
\hline Diabetes melito & 97 & 25,3 & $21,1-30,0$ & 27,8 & 4,2 & $2,0-9,0$ & 0,00003 \\
\hline Angina pós-infarto agudo do miocárdio & 69 & 18,0 & $14,3-22,3$ & 20,3 & 1,0 & $0,5-2,0$ & NS \\
\hline Reinfarto & 9 & 2,3 & $1,1-4,6$ & 44,4 & 3,2 & $0,7-15,2$ & NS \\
\hline Isquemia recorrente & 72 & 18,8 & $15,0-23,1$ & 22,2 & 1,4 & $0,7-2,7$ & NS \\
\hline Taquicardia ventricular & 17 & 4,4 & $2,7-7,1$ & 52,6 & 4,8 & $1,7-13,4$ & 0,001 \\
\hline Fibrilação ventricular & 17 & 4,4 & $2,7-7,1$ & 76,5 & 14,8 & $4,3-63,7$ & 0,00000 \\
\hline Uso de ácido acetilsalicílico & 332 & 86,5 & $82,5-89,6$ & 14,2 & 0,1 & $0,03-0,2$ & 0,000000 \\
\hline Uso de inibidor da ECA & 243 & 63,3 & $58,2-68,1$ & 14,8 & 0,5 & $0,3-0,8$ & 0,004 \\
\hline Uso de betabloqueador & 188 & 49,0 & $43,9-54,1$ & 8,0 & 0,2 & $0,1-0,4$ & 0,000000 \\
\hline
\end{tabular}

* OR de óbito na presença da complicação versus a ausência/não relato ou OR de óbito do uso da intervenção versus o não uso. 
Modelo logístico (modelo 3) para estudo do risco de óbito hospitalar em infarto agudo do miocárdio

na amostra de prontuários.

\begin{tabular}{|c|c|c|c|c|c|}
\hline Variável & Parâmetro & Erro padrão & $\begin{array}{c}\operatorname{Pr}>\chi^{2} \\
p\end{array}$ & OR & IC95\% \\
\hline Intercepto & $-1,468$ & 0,516 & 0,004 & - & - \\
\hline \multicolumn{6}{|l|}{ Faixa etária (em anos) } \\
\hline $61-80$ & 0,914 & 0,388 & 0,02 & 2,50 & $1,17-5,34$ \\
\hline$>80$ & 2,265 & 0,814 & 0,005 & 9,63 & $1,95-47,52$ \\
\hline \multicolumn{6}{|l|}{ Classe Killip à admissão } \\
\hline II & 0,649 & 0,678 & NS & 1,91 & $0,51-7,22$ \\
\hline III & 1,799 & 0,743 & 0,02 & 6,04 & $1,41-25,93$ \\
\hline IV & 3,277 & 1,129 & 0,004 & 26,50 & $2,90-242,11$ \\
\hline Diabetes & 0,859 & 0,392 & 0,03 & 2,36 & $1,09-5,09$ \\
\hline Eletrocardiograma não realizado/ignorado & 2,470 & 0,621 & 0,0001 & 11,82 & $3,50-39,92$ \\
\hline Taquicardia ventricular & 2,145 & 0,634 & 0,0007 & 8,54 & $2,47-29,59$ \\
\hline Fibrilação ventricular & 3,525 & 0,747 & 0,0001 & 33,97 & $7,85-146,93$ \\
\hline Isquemia recorrente & 0,987 & 0,429 & 0,02 & 2,68 & $1,16-6,22$ \\
\hline Uso de ácido acetilsalicílico & $-1,190$ & 0,485 & 0,01 & 0,30 & $0,12-0,79$ \\
\hline Uso de betabloqueador & $-1,173$ & 0,416 & 0,005 & 0,31 & $0,14-0,70$ \\
\hline Uso de inibidor da ECA & $-0,825$ & 0,394 & 0,04 & 0,44 & $0,20-0,95$ \\
\hline
\end{tabular}

-2 LOG L intercepto e covariáveis $=225,5 \cdot \chi^{2}$ para covariáveis $=164,9 \mathrm{com} 13 \mathrm{GL}(\mathrm{p}=0,0001)$.

Concordância $=90,1 \%$. ĉ (Hosmer-Lemeshow) $=6,49 \operatorname{com} 8 \mathrm{GL}(p=0,60)$.

precursores da doença coronariana isquêmica não apresentaram efeito significativo à modelagem, à exceção do diabetes, que se associou a uma maior chance de óbito.

Observou-se uma associação entre classe Killip à admissão crescente (refletindo um gradiente de disfunção ventricular) e um gradiente de risco crescente com o aumento da classe. Optou-se por manter Killip II no modelo, apesar de não ter significância estatística, por apresentar razão de chances próxima a dois, além de existir plausibilidade biológica de um maior risco de morte em relação à categoria de referência Killip I 14,15. Além disso, a inclusão da classe Killip II à categoria de referência não modificava as estimativas dos parâmetros do modelo final; apenas era perdida a informação da tendência a uma maior chance de morte associada à Killip II em comparação à Killip I.

Foram estudados os efeitos de diferentes padrões eletrocardiográficos de infarto agudo do miocárdio relatados (parede anterior: $39,3 \%$ e letalidade $=18,5 \%$; inferior: $33,6 \%$ e letalidade $=14,7 \%$; infarto agudo do miocárdio sem onda Q: $15,6 \%$ e letalidade $=11,7 \%$; bloqueio de ramo esquerdo: $3,1 \%$ e letalidade $=33,3 \%$ ). Em 24 pacientes $(6,3 \%)$ o eletrocardiograma não foi realizado (5) ou o resultado não estava relatado no prontuário (19); em oito casos $(2,1 \%)$ o eletrocardiograma foi realizado e o infarto agudo do miocárdio afastado. A letalidade associada aos casos com eletrocardiograma não realizado/ignorado foi muito alta $-75,0 \%$, com OR = 14,7 (IC95\%: 5,3-46,7; p = 0,0000000) em relação à simples realização de eletrocardiograma, não importando qual o padrão resultante. A variável eletrocardiograma não realizado/ignorado foi o único padrão eletrocardiogáfico que apresentou efeito independente na análise multivariada.

Em relação à presença de arritmia, tanto a taquicardia ventricular como a fibrilação ventricular durante a internação, estiveram associadas a uma chance elevada de óbito hospitalar na análise multivariada. Quanto à fibrilação ventricular, analisou-se, também, o efeito da fibrilação ventricular primária separadamente da secundária; ambas persistiram associadas a uma maior chance de óbito, mas o número reduzido de casos levava a estimativas pouco precisas e tornava o modelo mais instável.

A variável isquemia recorrente (angina pósinfarto agudo do miocárdio e/ou reinfarto) não era estatisticamente significativa na análise bivariada (Tabela 3 ), mas foi incluída na modelagem pela plausibilidade biológica de associação com maior risco de morte 15 , que ficou evidenciada no modelo 3 . 
As intervenções que apresentaram associação com menor chance de óbito foram o uso de ácido acetilsalicílico, de betabloqueador e de inibidores da ECA. A trombólise intravenosa não apresentou efeito significativo sobre o risco de óbito desde a análise bivariada $(\mathrm{OR}=0,6$; IC95\%: 0,3-1,3).

Inicialmente, o uso de cateterismo cardíaco foi incluído no modelo estudado a partir do prontuário médico. Desde o primeiro modelo aplicado baseando-se na AIH (modelo 1, Tabela 2), observou-se uma associação significativa e consistente do cateterismo com menor chance de óbito durante a internação por infarto agudo do miocárdio, que se manteve nos modelos a partir do prontuário, apesar da freqüência relativamente baixa do procedimento. Entretanto, o cateterismo por si só não é uma intervenção terapêutica e, sim, diagnóstica. Na amostra ocorreu apenas um óbito no grupo submetido a cateterismo, sendo que este caso comportava-se como influencial e sua retirada tornava o modelo a partir do prontuário instável. Optou-se pela exclusão da variável cateterismo cardíaco do modelo 3 (Tabela 4), observando-se que a sua exclusão não modificava de forma significativa o desempenho do modelo nem as estimativas dos parâmetros.

No processo de modelagem, outras variáveis disponíveis na amostra de prontuário não alcançaram significância estatística ou mesmo um efeito importante expresso por meio de uma razão de chances elevada.

Foram estudadas também variáveis correspondentes a aspectos da estrutura e movimento dos hospitais, a partir da hipótese de que a composição de algumas dessas variáveis poderia refletir perfis hospitalares diferentes. Foram estudados a natureza jurídica dos hospitais, o total de leitos e a participação do Sistema Integrado de Procedimentos de Alta Complexidade (SIPAC - dados obtidos do Cadastro de Hospitais do SIH/SUS referente a 1997), assim como foram obtidas informações sobre a existência de hemodinâmica e de emergência diretamente das unidades hospitalares. Tais variáveis não alcançaram significância estatística nem acrescentaram informação adicional ao modelo de variação de risco de óbito hospitalar.

Os testes para interação entre variáveis com indicação biológica para tal não sugeriram a sua presença (foram sistematicamente não significativos). Foi investigada a existência de interação entre faixa etária e as diversas variáveis que expressam gravidade e uso de intervenções, entre diabetes e classe Killip, diabetes e isquemia recorrente, classe Killip e taquicardia ventricular, classe Killip e fibrilação ventricular, uso combinado de intervenções, entre outras. Apesar do sexo não ter sido uma variável significativa, também foi investigada a possibilidade de interação com outras variáveis.

O diagnóstico do modelo foi feito valendose da análise dos resíduos e do impacto da retirada de observações muito desviantes sobre os modelos.

A Figura 1 mostra as curvas ROC das probabilidades preditas de cada um dos três modelos, construídas a partir da determinação da sensibilidade e especificidade para cada ponto de corte do valor predito em relação ao valor observado. Nela estão descritos, também, os resultados do cálculo das áreas sob cada uma das curvas ROC, com o respectivo erro padrão entre parêntesis. Não foi detectada diferença significativa entre as áreas sob as curvas ROC dos modelos desenvolvidos a partir das variáveis presentes na $\mathrm{AIH}$, originais ou corrigidas. Portanto, apesar das limitações quanto à precisão de algumas variáveis na AIH, o desempenho relativo entre os dois modelos (o modelo das variáveis originalmente digitadas na $\mathrm{AIH}$ versus corrigidas pelo prontuário) não foi diferente quando analisado por meio das respectivas curvas ROC.

Já a comparação da área sob a curva ROC do modelo 3 (a partir do prontuário) com cada uma das áreas das curvas ROC dos modelos $2 \mathrm{e}$ 3 mostrou uma diferença significativa, $\operatorname{com} \mathrm{p}=$ 0,0000 a favor de um melhor desempenho do a partir do prontuário médico. Portanto, a inclu-

Figura 1

Curvas ROC das probabilidades preditas pelos modelos estudados.

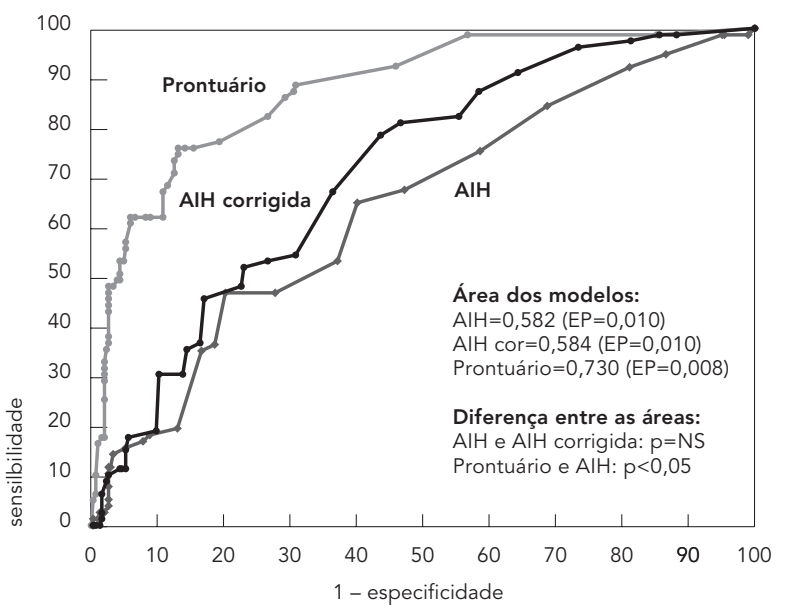


são de variáveis de gravidade e de uso de intervenções que não estavam disponíveis na AIH melhorou muito o ajuste e a sensibilidade e especificidade do modelo, como era esperado.

Durante o processo de modelagem aplicouse ainda o modelo 1 para o banco da AIH do Município do Rio de Janeiro como um todo; o desempenho desse modelo não diferiu de forma significativa em relação ao modelo a partir da amostra de AIH; a curva ROC a ele correspondente situou-se em posição intermediária entre as curvas dos modelos 1 e 2 .

\section{Discussão}

O Sistema da AIH teve sua origem nos anos sessenta para padronizar o pagamento dos serviços prestados como tabela de honorários médicos 8. Atualmente, cobre a produção hospitalar de todos os serviços financiados pelo setor público e é amplamente disponibilizado pelo Departamento de Informação e Informática do SUS (DATASUS, Ministério da Saúde). Apesar de surgir dentro da lógica contábil do pagamento da atenção hospitalar, gera, também, informações sobre a morbidade hospitalar, o consumo de recursos, o uso de procedimentos diagnósticos e de alta complexidade, características demográficas e geográficas, natureza e complexidade dos hospitais. Portanto, o SIH/ SUS pode constituir um instrumento valioso para o planejamento, a avaliação da qualidade e estudos epidemiológicos.

Autores como Wray et al. 16 têm discutido questões conceituais relativas à utilização de bancos de dados administrativos na avaliação da qualidade da assistência. A grande vantagem do uso dessas bases está na sua rápida disponibilidade e conseqüente economia de tempo e recursos. Além disso, elas podem refletir a realidade da prática diária de uma forma mais transparente do que os ensaios clínicos randomizados ou mesmo estudos observacionais prospectivos, que geralmente são realizados em serviços diferenciados. Entretanto, o valor das informações obtidas por meio dessas bases tem sido questionado, em virtude da inadequação dos dados clínicos nelas contidos e, conseqüentemente, da incapacidade de controlar adequadamente a variação dos pacientes. Tem sido crescente a utilização, por parte dos hospitais, de suas bases de dados administrativos para monitorar indicadores como taxas de mortalidade, tempos de permanência e taxas de readmissão. No entanto, tem havido resistência a usá-las na comparação de resultados entre diferentes hospitais, sobretudo a partir da hipótese de que seria a variação não mensurada na gravidade dos casos à admissão a principal explicação para resultados desfavoráveis em número excessivo, e não uma atenção de pior qualidade em relação a padrões estabelecidos. Mas alguns estudos têm mostrado que a gravidade não explica toda a variação observada entre as mortalidades de diferentes hospitais e que diferenças residuais refletem a qualidade da assistência 17.

Artigo nosso anterior discutiu a pertinência do uso da AIH na avaliação da qualidade da assistência ao infarto agudo do miocárdio 11, documentando a qualidade satisfatória do diagnóstico de infarto agudo do miocárdio da $\mathrm{AIH}$ e a precisão em geral satisfatória das variáveis demográficas (sexo, faixa etária), de processo (uso de procedimentos e intervenções) e de resultado (óbito, motivo da saída). A precisão das variáveis demográficas e de resultado foi superior a das variáveis de processo. Entre as variáveis de processo estudadas, a precisão do uso de cirurgia de revascularização miocárdica, de cateterismo cardíaco e de angioplastia coronária foi superior à precisão do uso de trombólise intravenosa e de UTI/UC. O elevado sub-registro do diagnóstico secundário na AIH foi a maior limitação observada. Travassos-Veras \& Martins 10 também relataram esta limitação referindo-se à freqüência reduzida do registro e ao fato de estar limitado a apenas um diagnóstico secundário.

O diagnóstico secundário adequadamente informado poderia fornecer dados para um melhor ajuste de gravidade, que foi um dos fatores principais para explicar a diferença de desempenho entre os modelos da AIH versus prontuário neste estudo. Outro fator limitante da $\mathrm{AIH}$ é a disponibilidade limitada quanto a variáveis de processo da assistência. No caso do infarto agudo do miocárdio, o único fármaco disponível é a estreptoquinase (droga usada para a trombólise intravenosa), além disso, conta apenas com a informação sobre internação em UTI/UC, uso de cateterismo cardíaco, angioplastia coronariana e cirurgia cardíaca.

A diferença entre as naturezas jurídicas encontradas na análise baseando-se na AIH desapareceu na análise a partir do prontuário, que permitiu um melhor ajuste da gravidade e do processo. A maior chance de óbito associada às naturezas jurídicas privada e universitária na modelagem com base na AIH (modelos 1 e 2, Tabela 2) desapareceu no modelo a partir do prontuário (modelo 3, Tabela 4) após a introdução de variáveis que expressavam gravidade e o uso de intervenções. Entretanto, perfis podem ser eventualmente identificados, refletin- 
do padrões de gravidade e/ou processo diferenciado.

De uma forma geral, a letalidade de $20,6 \%$ evidenciada nesta amostra, distancia-se bastante das cifras reduzidas que são alcançadas em ensaios clínicos randomizados 18 ou centros selecionados 19,20, ou estimadas a partir da projeção da eficácia das tecnologias disponíveis para tratamento dos casos 21. Entretanto, outros estudos internacionais de casos não selecionados têm relatado letalidades hospitalares elevadas associadas ao infarto agudo do miocárdio, variando de $18,0 \%$ a $21,7 \%$ 22,23,24,25. Dois estudos nacionais de casos com base na AIH relataram letalidade de $17,1 \%$ no Estado de São Paulo ${ }^{9}$ e 18,4\% no Estado do Rio de Janeiro 5; outro estudo mostrou letalidade de 30 dias de 22,0\% em um hospital universitário de Botucatu, São Paulo 26.

A estrutura de sexo e faixa etária da amostra, a mediana de delta tempo de 12 horas e a prevalência de diabetes foram semelhantes às relatadas por outros estudos nacionais 4,5,20,21,26. O percentual de Killip I à admissão observado foi muito superior aos $56,0 \%$ relatados por Brown et al. 25 e mais próximo dos $77,8 \%$ de Passos et al. 20. A incidência de 1,8\% de fibrilação ventricular primária foi menor que a de outro estudo $(4,7 \%) 27$.

Em relação ao processo de assistência na amostra, ressalta-se a subutilização de algumas intervenções com eficácia documentada por evidência científica, destacando-se os trombolíticos, betabloqueadores e ácido acetilsalicílico. Já o uso de inibidores da ECA foi bem difundido (63,3\%), semelhante ou maior do que em outros estudos 18,26. Quanto à utilização de UTI/UC, alguns estudos têm relatado de 70,0\% a $90,0 \% 23,24,25$.

A não realização de eletrocardiograma ou a ausência de informação sobre seu resultado no prontuário (eletrocardiograma não realizado/ignorado) esteve consistentemente associada a uma letalidade elevada e foi o único padrão eletrocardiográfico que apresentou efeito independente na análise multivariada. Esta variável foi incluída enquanto um possível indicador de problemas de assistência ou de qualidade da informação. A variação do uso de cateterismo dependeu da disponibilidade de hemodinâmica no hospital da AIH; estudos internacionais têm relatado freqüências variadas desse uso durante a internação do infarto agudo do miocárdio, em geral maiores que os 13,3\% da amostra 18,24.

Na modelagem foram avaliados dois tipos de modelos: o logístico e o hierárquico. A possibilidade de existência de correlação interna no nível dos hospitais foi investigada por meio de modelagem hierárquica, que não detectou efeito randômico significativo. Isto pode estar relacionado ao tamanho da amostra, tanto do ponto de vista de número de observações do nível superior (os hospitais), como do número de observações do primeiro nível (o caso), uma vez que o cálculo da amostra tomou como base a proporção de óbitos no município como um todo. De qualquer forma, a não detecção de efeito randômico ratificou a opção pelo modelo logístico.

As associações independentes encontradas entre faixa etária mais elevada, diabetes e classe Killip crescente à admissão são consistentes com a literatura 15. A variável Diagnóstico Secundário utilizada nos modelos da AIH perdeu sua importância no modelo derivado a partir do prontuário, frente à possibilidade de controle dos vários fatores de risco, gravidade e complicações, separadamente. Destes, os únicos que apresentaram efeito significativo sobre a letalidade foram: classe Killip $\geq 2$, diabetes, taquicardia ventricular e fibrilação ventricular.

As intervenções que apresentaram associação com menor chance de óbito foram o uso de ácido acetilsalicílico, de betabloqueador e de inibidores da ECA. O uso de trombólise não apresentou significância estatística desde a análise bivariada, o que pode relacionar-se ao tamanho da amostra. Todas essas intervenções têm eficácia comprovada em reduzir a mortalidade no infarto agudo do miocárdio, sendo a da trombólise a maior 2. Entretanto, a freqüência de uso do ácido acetilsalicílico, betabloqueador e inibidores da ECA foi bastante superior à da trombólise, o que pode explicar, pelo menos em parte, a não documentação do efeito desta última na amostra.

Considerou-se também a possibilidade de interferência do chamado confundimento por indicação ("confounding by indication"), que ocorre quando se analisa o efeito de intervenções fora de ensaios clínicos controlados e surge em conseqüência ao fato de que as indicações para um determinado tratamento são quase sempre indicadoras de risco especial 28,29. Neste estudo, adotou-se a estratégia de controle na análise por meio da inclusão, no modelo, de variáveis relacionadas à gravidade do caso e à indicação das intervenções, como proposto por alguns autores 28 . Assim, alguns dos principais possíveis fatores de confundimento relacionados à indicação do ácido acetilsalicílico, betabloqueadores e inibidores da ECA foram controlados durante o processo da modelagem.

Uma possível influência da confirmação do diagnóstico de infarto agudo do miocárdio tam- 
bém foi investigada, baseando-se na hipótese de que os casos de infarto agudo do miocárdio afastado ou ignorado poderiam interferir no ajuste dos modelos ou introduzir problemas de especificação. Entretanto, não só o percentual desses casos foi reduzido como a confirmação (ou não) do diagnóstico não apresentou significância estatística nem modificou nenhum dos modelos estudados.

Durante o processo de modelagem, analisou-se, inicialmente, a influência de cada categoria de ignorado quanto à informação presente no prontuário sobre as diversas variáveis, criando-se "dummies" para a presença, a $a u$ sência e o ignorado para cada complicação ou intervenção. A categoria ignorado foi sistematicamente não significativa nos vários modelos testados e a sua exclusão não modificava o modelo; a única exceção foi o eletrocardiograma não realizado/ignorado, onde o efeito principal era do ignorado, mais freqüente que o não realizado. Assim, optou-se por incluir o ignorado na categoria de referência. Apesar desta decisão poder ser fonte de viés, o mesmo seria conservador quanto ao efeito estudado.

Os modelos estatísticos utilizados neste estudo podem ser considerados testes com a finalidade de estimar a probabilidade de ocorrência de um evento, no caso, óbito hospitalar. Uma aplicação a ser investigada é a utilização desses modelos para a predição de óbitos não esperados, ou seja, óbitos ocorridos em casos com baixa probabilidade estimada pela modelagem. Tais óbitos poderiam ser investigados como possíveis eventos-sentinela de problemas de qualidade da assistência. Nesse sentido, o modelo seria útil para triagem de óbitos não esperados a serem investigados posteriormente 6 .

\section{Conclusões}

O prontuário foi superior à AIH no estudo dos fatores associados à variação do risco de óbito hospitalar no infarto agudo do miocárdio, oferecendo uma melhor possibilidade de controle da gravidade dos casos e do processo da assis- tência. Diabetes, faixa etária elevada, classe Killip, isquemia recorrente, taquicardia ventricular e fibrilação ventricular associaram-se de forma independente a uma chance aumentada de óbito hospitalar. Houve associação entre o uso de ácido acetilsalicílico, betabloqueadores e inibidores da ECA com uma menor chance de óbito hospitalar. Apesar da letalidade geral ter sido alta, o perfil de gravidade, principais fatores de risco e a freqüência das complicações estudadas foram semelhantes ou mais favoráveis do que em outros estudos. Como houve subutilização de intervenções eficazes, é possível esperar melhores resultados da assistência hospitalar ao infarto agudo do miocárdio no município estudado.

Apesar das limitações do banco da AIH, a correção de possíveis erros de digitação e informação encontrada não modificou substancialmente o ajuste do modelo construído com base nesse sistema. A confirmação do diagnóstico de infarto agudo do miocárdio e a precisão das variáveis da AIH foram satisfatórias; a maior limitação foi o registro extremamente reduzido do diagnóstico secundário. Um melhor preenchimento dessa variável melhoraria a possibilidade de ajustar a gravidade dos casos.

A limitação do modelo a partir da AIH em explicar a variação do risco de óbito hospitalar na amostra, relacionou-se à disponibilidade restrita de variáveis referentes à gravidade dos casos e às intervenções farmacológicas utilizadas na assistência ao infarto agudo do miocárdio.

A constatação de que a qualidade das informações disponíveis no SIH/SUS é melhor do que a corriqueiramente imputada a este banco, pelo menos quanto ao infarto agudo do miocárdio no Município do Rio de Janeiro, aponta para a pertinência do seu uso como um instrumento de avaliação da qualidade da assistência hospitalar. Apesar das limitações inerentes ao fato de ser um banco administrativo, tem a grande vantagem de estar amplamente implantado e disponibilizado no país. É importante a avaliação crítica de suas potencialidades e limitações com respeito ao uso aplicado à área da avaliação da assistência hospitalar a este e outros agravos. 


\section{Resumo}

O objetivo deste estudo é avaliar o desempenho do Sistema de Informações Hospitalares (SIH) em relação ao prontuário médico na análise dos fatores associados à variação do risco de óbito hospitalar no infarto agudo do miocárdio. O estudo envolveu uma amostra aleatória, estratificada por hospital, de 391 prontuários médicos sorteados com base nos 1.936 formulários de Autorização de Internação Hospitalar (AIH) registrados com o diagnóstico principal de infarto agudo do miocárdio no Município do Rio de Janeiro, Brasil, em 1997. Para estudo dos fatores associados à variação do risco de óbito hospitalar foram usados modelos logísticos a partir do SIH e do prontuário, com construção de curvas ROC para comparar desempenho relativo entre eles. O diagnóstico foi confirmado em 91,7\% dos casos; a letalidade foi 20,6\%. O modelo desenvolvido a partir do prontuário apresentou o melhor ajuste por incluir variáveis de gravidade e processo não disponíveis no SIH (concordância = 90,1\%). O modelo derivado do SIH teve um menor poder explicativo (concordância $=70,6 \%)$, mas a correção de erros de digitação e informação através do prontuário não modificou significativamente seu desempenho. A maior limitação do SIH foi o elevado sub-registro do diagnóstico secundário.

Sistemas de Informação; Infarto do Miocárdio; Mortalidade Hospitalar; Registros Médicos

\section{Colaboradores}

C. C. Escosteguy participou da elaboração do desenho do estudo, do trabalho de campo, da análise de dados e da redação do texto. M. C. Portela orientou o estudo, participou da análise dos dados e da revisão dos resultados e do texto. R. A. Medronho participou do trabalho de campo, da análise dos dados e da revisão dos resultados e do texto. M. T. L. Vasconcellos participou da elaboração do desenho da amostra e da análise estatística.

\section{Referências}

1. Lolio CA, Lotufo PA, Lira AC, Zanetta DMT, Massad E. Tendência da mortalidade por doença isquêmica do coração nas capitais de regiões metropolitanas do Brasil, 1979-89. Arq Bras Cardiol 1995; 64:213-6.

2. American College of Cardiology/American Heart Association Task Force Report. 1999 update: guidelines for the management of patients with acute myocardial infarction. Guideline. J Am Coll Cardiol 1999; 34:890-911.

3. Alexander RW, Pratt CM, Roberts R. Diagnosis and management of patients with acute myocardial infarction. In: Alexander RW, Schlant RC, Fuster V, editors. Hurst's the heart: arteries and veins. $9^{\text {th }}$ Ed. New York: McGraw-Hill; 1998. p. 1345-433.

4. Escosteguy CC, Medronho RA, Portela MP. Avaliação da letalidade hospitalar do infarto agudo do miocárdio no Estado do Rio de Janeiro através do uso do Sistema de Informações Hospitalares/ SUS. Cad Saúde Colet (Rio J) 1999; 7:39-59.

5. Escosteguy CC, Portela MC, Medronho RA, Vasconcellos MTL. Pharmacological management of acute myocardial infarction in the municipal district of Rio de Janeiro. Rev Paul Med 2001; 119: 193-9.

6. Escosteguy CC. Avaliação da qualidade da assistência hospitalar ao infarto agudo do miocárdio no Município do Rio de Janeiro [Tese de Doutorado]. Rio de Janeiro: Escola Nacional de Saúde Pública, Fundação Oswaldo Cruz; 2000.

7. Travassos-Veras CM. Equity in the use of private hospitals contracted by a complusory insurance scheme in the city of Rio de Janeiro, Brazil, in 1986 [Ph.D. Thesis]. London: Department of Social Sciences and Public Administration, The London School of Economics and Political Sciences; 1992.

8. Leucovitz E, Pereira TRC. SIH/SUS (Sistema AIH): 1983-1991. Série Estudos em Saúde Coletiva 1993; 57:1-83.

9. Marcopito LF, Goldfeder AJ, Schenkman S. Infarto agudo do miocárdio no Estado de São Paulo. Mortes ocorridas em Hospitais, de 1979 a 1996, e Letalidade Hospitalar no SIH/SUS, de 1984 a 1998. Arq Brasil Cardiol 2000; 74:39-42.

10. Travassos-Veras CM, Martins MS. A confiabilidade dos dados nos formulários de Autorização de Internação Hospitalar (AIH), Rio de Janeiro, Brasil. Cad Saúde Pública 1994; 10:339-55.

11. Escosteguy CC, Portela MC, Medronho RA, Vasconcellos MTL. O Sistema de Informações Hospitalares e a assistência ao infarto agudo do miocárdio. Rev Saúde Pública 2002; 36:491-9.

12. Tunstall-Pedoe H, Kuulasmaa K, Amouyel P, Arveiler D, Rajakangas AM, Pajak A. Myocardial infarction and coronary deaths in the World Health Organization MONICA Project. Registration procedures, event rates, and case-fatality rates in 38 populations from 21 countries in four continents. Circulation 1994; 90:583-612.

13. Fletcher RH, Fletcher SW, Wagner EH. Clinical epidemiology: the essentials. 3rd Ed. Baltimore: Williams \& Wilkins; 1996. 
14. Killip T, Kimball JT. Treatment of myocardial infarction in a coronary care unit: a two experience with 250 patients. Am J Cardiol 1967; 20:457-64.

15. Antman EM, Braunwald E. Acute myocardial infarction. In: Braunwald E, editor. Heart disease. A textbook of cardiovascular medicine. $5^{\text {th }}$ Ed. Philadelphia: WB Saunders; 1997. p. 1184-288.

16. Wray NP, Ashton CM, Kuykendall DH, Hollingsworth JC. Using administrative databases to evaluate the quality of medical care: a conceptual framework. Soc Sci Med 1995; 40:1707-15.

17. Iezzoni LI. The risks of risk adjustment. JAMA 1997; 278:1600-7.

18. Pilote L, Califf RM, Sapp S, Muller DP, Mark DB, Weaver WD, et al. Regional variation across the United States in the management of acute myocardial infarction. N Engl J Med 1995; 333:56572.

19. Scott IA, Eyeson-Annan ML, Huxley SL, West MJ. Optimising care of acute myocardial infarction: results of a regional quality improvement project. J Qual Clin Pract 2000; 20:12-9.

20. Passos LCS, Lopes AA, Souza LAM, Soza JAA, Souza Neto JP, Souza Filho J, et al. Preditores da mortalidade hospitalar na era trombolítica para o infarto agudo do miocárdio em Salvador (BA). Arq Brasil Cardiol 1997; 68:249-55.

21. Silva LK, Escosteguy CC, Machado CV. Metodologia para a estimativa de padrões de qualidade: o caso do infarto agudo do miocárdio. Cad Saúde Pública 1996; 12 Suppl 2:71-83.

22. Every NR, Frederick PD, Robinson M, Sugarman J, Bowlby L, Barron HV. A comparison of the National Registry of Myocardial Infarction 2 with the cooperative cardiovascular project. J Am Coll Cardiol 1999, 33:1886-94.
23. Reikvam A, Aursnes I. Hospital mortality from acute myocardial infarction has been modestly reduced after introduction of thrombolytics and aspirin: results from a new analytical approach. European Secondary Prevention Study Group. J Clin Epid 1999; 52:609-13.

24. Mahon NG, O'Rorke C, Codd MB, McCann HA, McGarry K, Sugrue DD. Hospital mortality of acute myocardial infarction in the thrombolytic era. Heart 1999; 81:478-82.

25. Brown N, Young T, Gray D, Skene AM, Hampton JR. Inpatient deaths from acute myocardial infarction, 1982-92: analysis of data in the Nottingham Heart Attack Register. BMJ 1997; 315:159-64.

26. Zornoff LAM, Paiva SAR, Assalin VM, Póla PMS, Becker LE, Okoshi MP, et al. Perfil clínico, preditores de mortalidade e tratamento de pacientes após infarto agudo do miocárdio, em hospital terciário universitário. Arq Brasil Cardiol 2002; 78: 396-400.

27. Thompson CA, Yarzebski J, Goldberg RJ, Lessard D, Gore JM, Dalen JE. Changes over time in the incidence and case-fatality rates of primary ventricular fibrillation complicating acute myocardial infarction: perspectives from the Worcester Heart Attack Study. Am Heart J 2000; 139:1014-21.

28. Poses RM, Smith WR, McClish DK, Anthony M. Controlling for confounding by indication for treatment. Are administrative data equivalent to clinical data? Med Care 1995; 33:AS36-46.

29. Miettinen OS, Cook EF. Confounding: essence and detection. Int J Epidemiol 1981; 114:593-603.

Recebido em 25/Mai/2004

Versão final reapresentada em 10/Fev/2005

Aprovado em 14/Fev/2005 